\title{
Katrina Brown
}

\section{Translucent Surface / Quiet Body}

Choreographic Notes

I NSTALL I N G

Working with a large table-like construction, a performing body is documented

simultaneously from above and below, while moving-and-drawing upon and over the glass table top. The $2 \mathrm{~m} \times 2 \mathrm{~m}$ elevated platform functions as a hard smooth ground for the performer and as a viewing screen for the documenting camera below.

Tracing paper $90 \mathrm{gms}$ operates between glass and skin as translucent membrane and screen, physically connecting and visually obscuring above and below. Natural daylight comes from two sides creating a light box effect with the table and tracing paper and accentuating the grey bare installation.

The two viewing perspectives (above and below) act as a visual annotation of one another, each offering distinct information of the gravitational forces at play and the reciprocal touch between surfaces of paper-glass and body-skin: making visible some of what is obscured from view on the other side of the screen.

The installation makes possible a live happening and digital documenting of a body's moving mass from beneath the surface.

These still pages lay out observations of the unfolding drawing processes and present a choreographics of forces, surfaces, body and documents.

Translucent Surface/Quiet Body is developing in residency at L'Animal à L'esquena, Celrà, Catalonia.
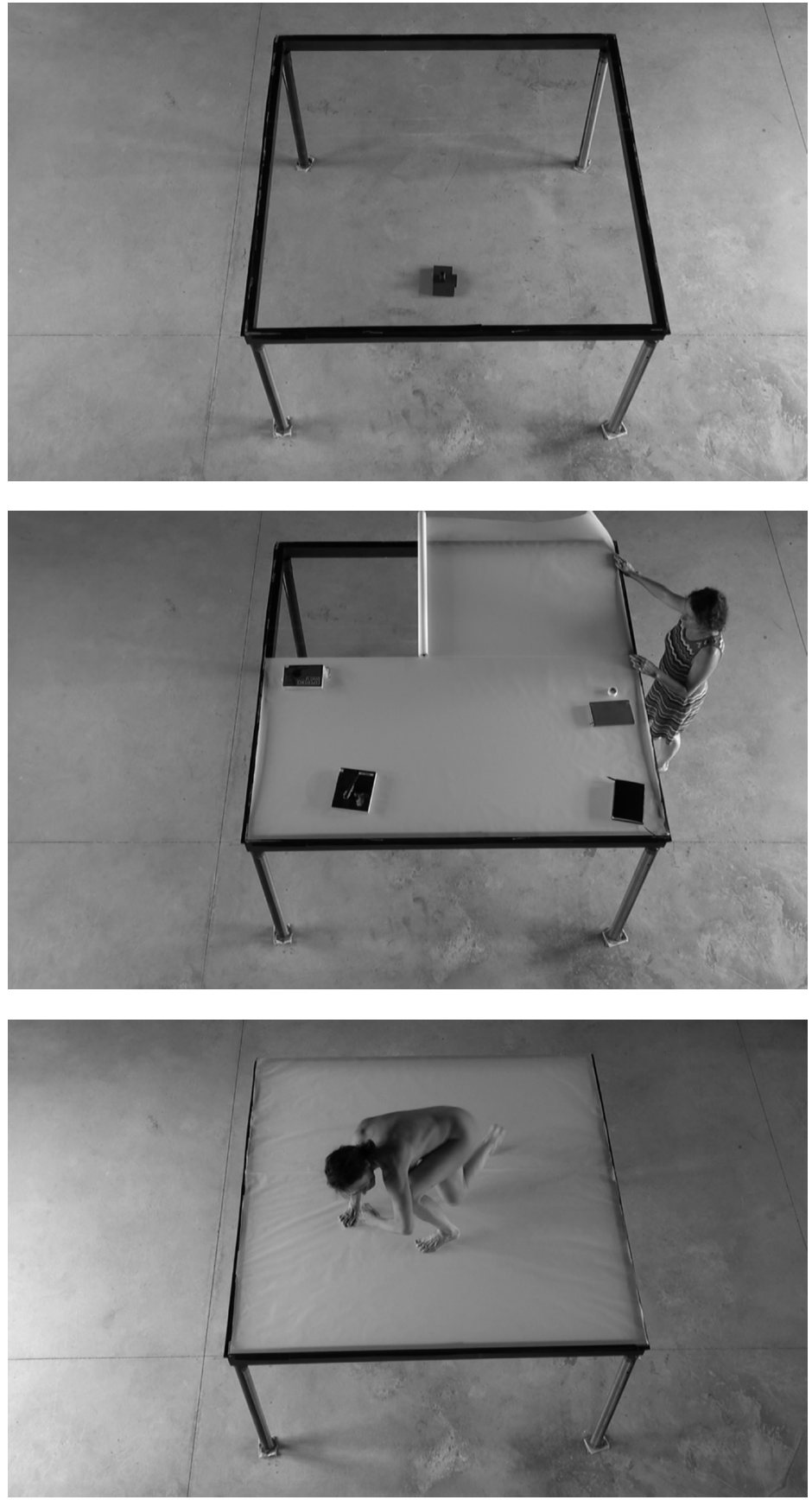


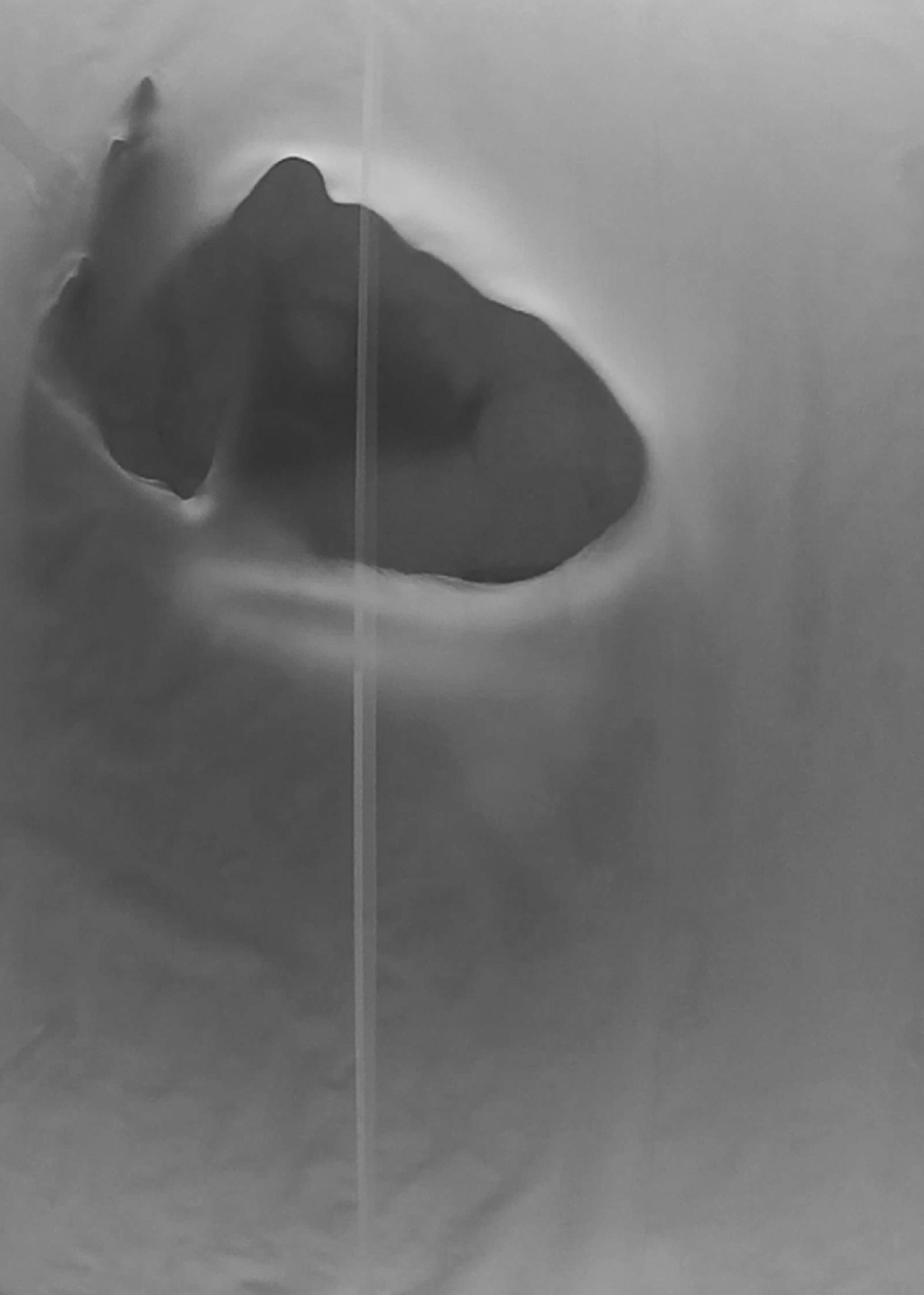




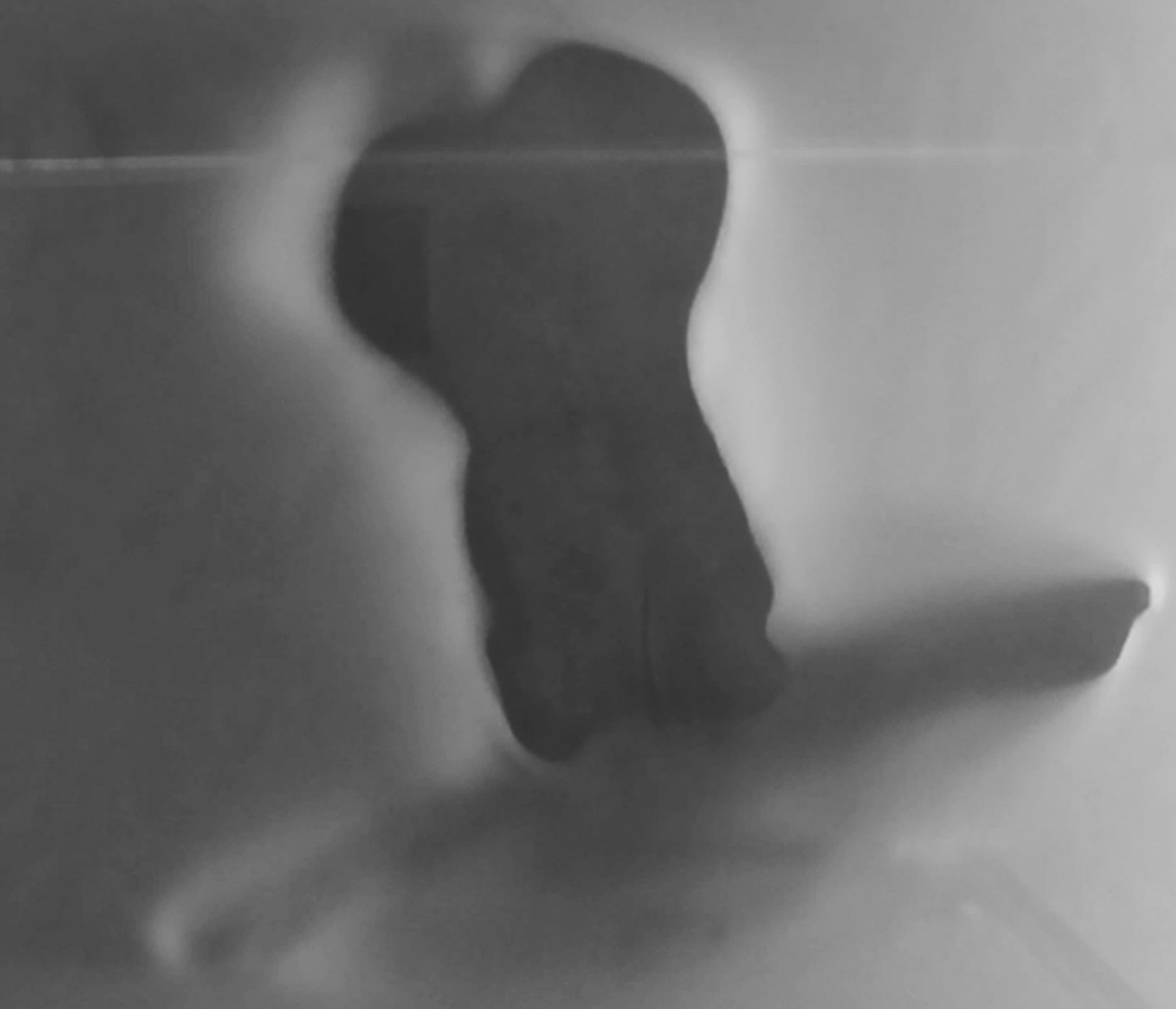




\section{SEEING, SENSING}

A not-usually seen perspective and mediation of the body opens up as the impact of the glass surface on the body's flesh becomes evident on the video documentation in the form of dark, flattened, creased areas, that appear, transform, disappear. Video stills capture, frame and suspend these flat impressions: gradations of darkness being proportional to amount of pressure, while a light-shadow reminds the viewer of the body's volume moving on her glass ground on the other side of the screen.

Capacities of surface as support, receptor, membrane, skin, page and screen coincide.

She becomes adept at moving within the edges of the $2 m \times 2 m$ surface area. She leans her weight and presses down on parts of the body (lower arm, shoulder blades, shins) so other parts of the body can lift. She twists, extends, curls and reconfigures her body around these fixed points of rotation. At the same time she works on softening her body and opening her skin to meet the hard smooth surface. She touches the paper and she feels the glass. Within these conditions she accelerates and slows her movement. Stilling her body, she notices miniscule shifts and drops of weight and touch. She senses the isolated quiet space she inhabits. Spiralling to stand, there is an accentuated sense of uprightness through her feet and spine on the firm but elevated ground.

On the platform she moves continually between the gravitational pull and horizontal expansion of the surface. She observes the quietness and space opening around her.

Two surfaces touch: paper and skin. The translucency of the paper creates a visual and visceral interruption between above and below. An entangled logic emerges between what is felt by the performer and seen by the documenting camera. There appears a discrepancy between the material reality of weight, metal, body, glass, pressure, impact, collision and the blurry-edged mediated bodily presence on the video footage that suggests a space without sound, orientation, resistance, or solid ground.
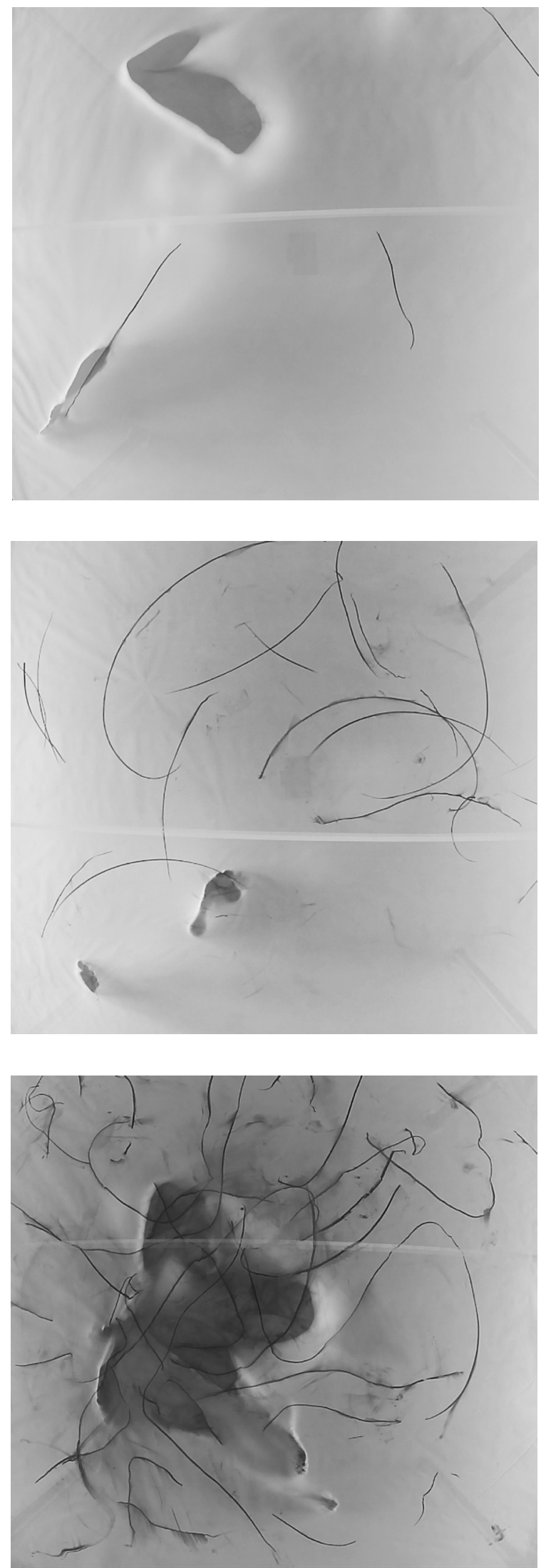

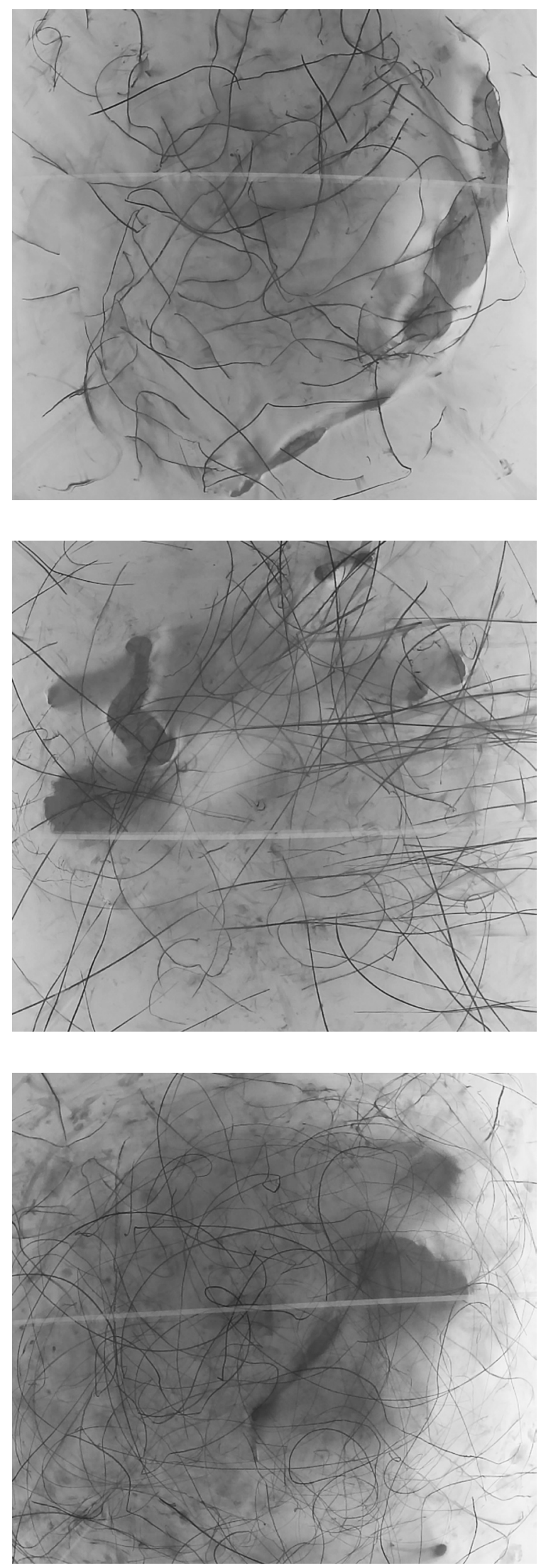

DRA WING, DOCUMENTING

She watches the video documentation of her body moving from beneath the paper-glass platform. She sees dark bodily shapes forming, transforming and unforming before her eyes and in any instant disappearing from view. She sees this drawing continually unfolding on the screen in front of her.

The documenting camera below captures two drawing processes: the moving mass of the performer's body over the surface and the performer's lining and unintentional smudging with charcoal. The moving body itself leaves no material mark on the surfaces of the installation in its pressing and being pressed, but the fleeting impressions are digitally captured. In contrast, the charcoal lines appear with pressure, intention, direction and remain on the paper. The lines appear on top of the performer rather than beneath her body, accentuating the collapsed graphic flat digital image. Lines simply start and end. Gesture and mark are visually interrupted.

Drawing is implemented and is operating as a choreographic activity between two materialities of body and surface. The documenting process opens up a logic of relations between body, surface, seeing, touching, vertical and horizontal.

\section{Absence is quietly present.}

Lines and smudges accumulate on and remain visible through the paper-glass translucent screen and a quietening darkening body presence recedes behind. The documentation from below shifts the visual dominance of the performer's actions by highlighting the impact of the surface on the body and quietly bringing the marked surface to the foreground.

She moves with increasing understanding of her situation and what is being generated for the documenting camera on the other side of the glass. She learns how fluidity, pressure, touch, withdrawal are being translated into visual data of dark, light, form and line. She becomes increasingly interested in how this affects her and allows her to investigate quietening body. 